\title{
Energy transfer in pure and rare-earth doped $\mathrm{SrAlF}_{5}$ crystals
}

\author{
S I Omelkov ${ }^{1,4}$, M Kirm ${ }^{2}$, V A Pustovarov ${ }^{1}$, L I Isaenko ${ }^{3}$ \\ ${ }^{1}$ Ural State Technical University, Mira 19, 620002 Yekaterinburg, Russia \\ ${ }^{2}$ Institute of Physics, University of Tartu, Riia 142, 51014 Tartu, Estonia \\ ${ }^{3}$ Institute of Geology and Mineralogy SB RAS, Russkaya 43, 630058 Novosibirsk, \\ Russia \\ E-mail: omelkovs@mail.ru
}

\begin{abstract}
This paper reports the results of investigation of luminescence and energy transfer processes in pure and Ce-, Gd- doped $\mathrm{SrAlF}_{5}$ single crystals by means of time-resolved UVVUV and XUV luminescence spectroscopy. Reliable evidence of self-trapped exciton (STE) formation was found. The complexity of STE emission and excitation bands points to the presence of several non-equivalent sites where excitons can localize. At temperatures above $\sim 150 \mathrm{~K}$ the STE emission is quenched, probably due to higher mobility and increasing probability of their interaction with defects. The role of the energy transfer of $\mathrm{Ce}^{3+}$ ions with lattice defects at different temperatures is discussed. For $\mathrm{Gd}^{3+}: \mathrm{SrAlF}_{5}$ crystal no effective direct excitation of the characteristic $3.97 \mathrm{eV} \mathrm{Gd}^{3+}$ emission was found. Instead, a new PL band at $5.6 \mathrm{eV}$ was revealed, which can be excited through ${ }^{8} \mathrm{~S}_{7 / 2}-{ }^{6} \mathrm{G}$ transitions in the $4 \mathrm{f}^{7}$ configuration of $\mathrm{Gd}^{3+}$. This phenomenon is tentatively explained by high probability of energy transfer from gadolinium to nearby defects. However, efficient excitation of the characteristic $\mathrm{Gd}^{3+}$ emission in the range of 7-11 eV points to resonant energy transfer from growth defects to the dopants in the same manner as proposed for $\mathrm{Ce}^{3+}$, and this process populates directly the ${ }^{6} \mathrm{P}_{7 / 2}$ level of $\mathrm{Gd}^{3+}$ from which emission takes place.
\end{abstract}

\section{Introduction}

$\mathrm{SrAlF}_{5}$ (SAF) crystals are promising candidates for the realization of all-solid-state lasers and other non-linear optical applications which operate in the ultraviolet and vacuum ultraviolet (VUV) spectral region. It has been shown that SAF is highly transparent down to the cutoff wavelength near $150 \mathrm{~nm}$ and its band gap was estimated to be $12 \mathrm{eV}[1,2]$. Vibrational spectroscopy studies of SAF were performed in [3]. However, the most interesting properties of this compound arise from its complex structure [4, 5]. All ions have several non-equivalent positions ( 4 for $\mathrm{Sr}^{2+}, 5$ for $\mathrm{Al}^{3+}$ and 20 for $\mathrm{F}^{-}$). The crystal field in these lattice positions varies, thus the dopants introduced to the various sites can have significantly different spectroscopic properties. This phenomenon offers wide material engineering capabilities, and the interest for such complex hosts is rapidly increasing (see for example [6]).

In our previous studies $[7,8]$ luminescence and energy transfer processes of pure and Ce-doped $\mathrm{SrAlF}_{5}$ crystals were analyzed. It was shown that $\mathrm{Ce}^{3+}$ ions substitute $\mathrm{Sr}^{2+}$ ions, which occupy 4 nonequivalent positions in the crystal lattice. Corresponding UV emission bands of Ce:SrAlF ${ }_{5}$ with nanosecond duration were assigned to $5 \mathrm{~d}-4 \mathrm{f}$ transitions of $\mathrm{Ce}^{3+}$ ions situated in different nonequivalent sites.

The present work continues this study, aimed at the deeper understanding of the mechanisms of energy transfer in $\mathrm{SrAlF}_{5}$ crystals, pure and doped with $\mathrm{Ce}^{3+}$ and $\mathrm{Gd}^{3+}$, and performed by means of time resolved luminescence spectroscopy at low temperatures under UV-VUV and extreme ultraviolet (XUV) excitation.

\footnotetext{
4 To whom any correspondence should be addressed.
} 
11th Europhysical Conference on Defects in Insulating Materials (EURODIM 2010)

IOP Publishing IOP Conf. Series: Materials Science and Engineering 15 (2010) 012011ｄoi:10.1088/1757-899X/15/1/012011

\section{Experimental}

\subsection{UV and VUV excitation}

Time-resolved and steady-state photoluminescence (PL) spectra in the region of $1.5-6.0 \mathrm{eV}$, timeresolved PL excitation spectra $(3.7-20.0 \mathrm{eV})$, and PL decay kinetics were recorded using synchrotron radiation excitation (SR) at the SUPERLUMI station (Beam-line I of HASYLAB at DESY) [9]. The studies were carried out in the temperature range of 8-300 K and the typical band-width of the exciting photon beam was $3 \AA$. A $0.3 \mathrm{~m}$ ARC Spectra Pro-308i spectrometer, used as detection system, is equipped both with a R6358P (Hamamatsu) photomultiplier and a CCD camera. Simultaneously with time-integrated luminescence, spectra were registered in a "slow" time window with the length of $\Delta \mathrm{t}=$ $35 \mathrm{~ns}$ and delayed by $\delta \mathrm{t}=123 \mathrm{~ns}$ relative to the exciting SR-pulse. The deconvolution method was used in analysis of the fast components of the PL decay kinetics. The time resolution of the whole detection system was 1 ns (FWHM).

\subsection{XUV excitation}

Time-integrated spectra $(2.5-8.0 \mathrm{eV})$ and decay curves of PL excited by XUV photons were recorded using the mobile luminescence setup [10] attached to the BW3 beamline of HASYLAB at DESY. PL spectra were measured by a $0.4 \mathrm{~m}$ vacuum monochromator (Seya-Namioka scheme) equipped with a microchannel plate photomultiplier (MCP 1645, Hamamatsu). No corrections of transmission of luminescence detection system were applied on PL emission spectra.

\subsection{Samples preparation}

All examined single crystals were grown using the Bridgman technique from the melt. $\mathrm{AlF}_{3}(99.99)$ and $\mathrm{SrF}_{2}$ (99.99) were taken as starting reagents. $\mathrm{CeF}_{3}$ or $\mathrm{GdF}_{3}$ (99.99) were added as dopants with concentration of 0.5 mass \%. However, the actual dopant concentration reached in samples can be lower. Details of the growth process and characterization of single crystals are presented in [5].

\section{Results}

As shown in [7,8], the luminescence of SAF comprises of several kind of emissions. Intrinsic luminescence and PL due to growth defects manifest themselves in all studied compounds. Intentional doping introduces emissions due to impurity ions, and the radiation-induced defect centers contribute in irradiated crystals. Most of these emissions are characterized by broad bands, which sometimes strongly overlap with each other. Hence, the analysis of luminescence processes in SAF crystals is not always straightforward and some features in excitation spectra could not be assigned to absorption by a specific luminescence center.

\subsection{Intrinsic luminescence and PL due to growth defects}

At $\mathrm{T}=10 \mathrm{~K}$ the most intensive band in PL spectra appears at $2.9 \mathrm{eV}$ (see figure 1). In [8] it is pointed out that it has weak fast nanosecond and strong microsecond duration decay components, whose maxima do not coincide exactly. Its PL excitation spectrum (figure 2, curve 1) consists of three bands in VUV region with identical positions of the maxima in both doped and pure SAF crystals. The position and shape of the PL band does not depend on the excitation energy within the range 7-11 eV.

Another band peaking at $4.5 \mathrm{eV}$ is found in the pure crystal and, less pronounced, in the Gd-doped compound. Its fast decay component has very short decay time (see inset in figure 1), and the excitation spectrum, as was already noted in [8], has opposite behavior to one recorded monitoring 2.9 $\mathrm{eV}$ emission (figure 2, curve 2). 


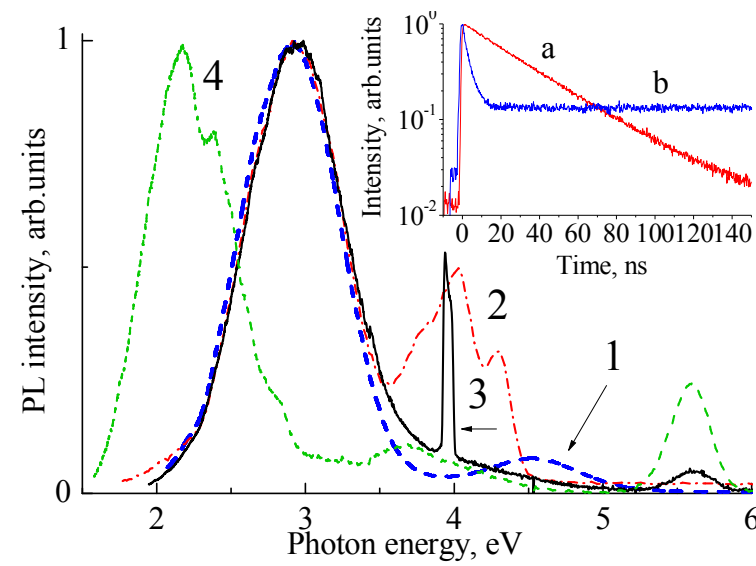

Figure 1. Time-integrated PL spectra of undoped and doped $\mathrm{SAF}$ at $\mathrm{T}=10 \mathrm{~K}$ : undoped $\mathrm{SAF}$, $E_{\text {exc }}=7.7 \mathrm{eV}$ from [8] (1); Ce:SAF, $E_{\text {exc }}=8.2 \mathrm{eV}$ from [7] (2); Gd:SAF, $E_{\text {exc }}=9 \mathrm{eV}$ (3) and $6.54 \mathrm{eV}$ (4). Inset: decay kinetics of Ce:SAF $\mathrm{E}_{\mathrm{exc}}=4.60$ $\mathrm{eV} \mathrm{E}_{\mathrm{em}}=3.85 \mathrm{eV}$ (a); and of undoped SAF $\mathrm{E}_{\mathrm{exc}}=7.7 \mathrm{eV} \mathrm{E} \mathrm{E}_{\mathrm{em}}=4.5 \mathrm{eV}$ from [8] (b).

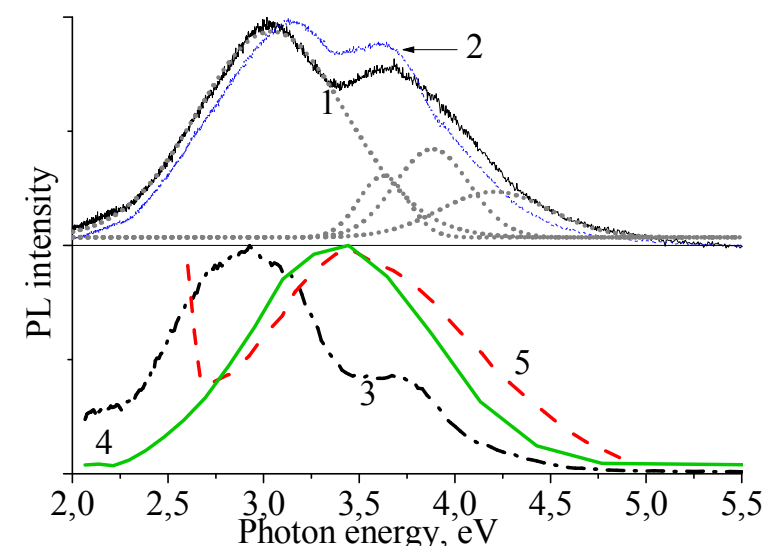

Figure 3. PL spectra of $\mathrm{SAF}$ at $\mathrm{T}=10 \mathrm{~K}$ : $E_{\text {exc }}=11.7 \mathrm{eV}$ undoped SAF (1); Gd:SAF (2); slow component of Ce:SAF emission at $\mathrm{E}_{\mathrm{exc}}=11.3 \mathrm{eV}(3) ; \mathrm{Pr}^{3+}: \mathrm{SAF} \mathrm{E}_{\mathrm{exc}}=11.17 \mathrm{eV}$ from [2] (4) and $\mathrm{Mn}^{3+}: \mathrm{SAF} \mathrm{E}_{\mathrm{exc}}=11.3 \mathrm{eV}$ from [11] (5). For curve (1) Gaussian fit is shown in gray.

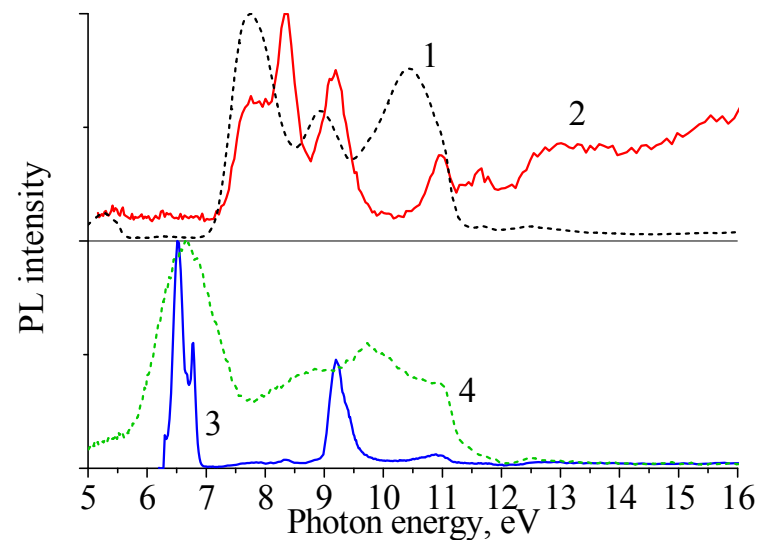

Figure 2. PL excitation spectra of various defect-related emissions at $\mathrm{T}=10 \mathrm{~K}$ : in undoped SAF from [8]: $E_{\mathrm{em}}=3 \mathrm{eV}(1), \mathrm{E}_{\mathrm{em}}=4.5$ $\mathrm{eV}$ (2); in Gd:SAF $\mathrm{E}_{\mathrm{em}}=5.6 \mathrm{eV}$ (3) and $\mathrm{E}_{\mathrm{em}}=2.02 \mathrm{eV}(4)$

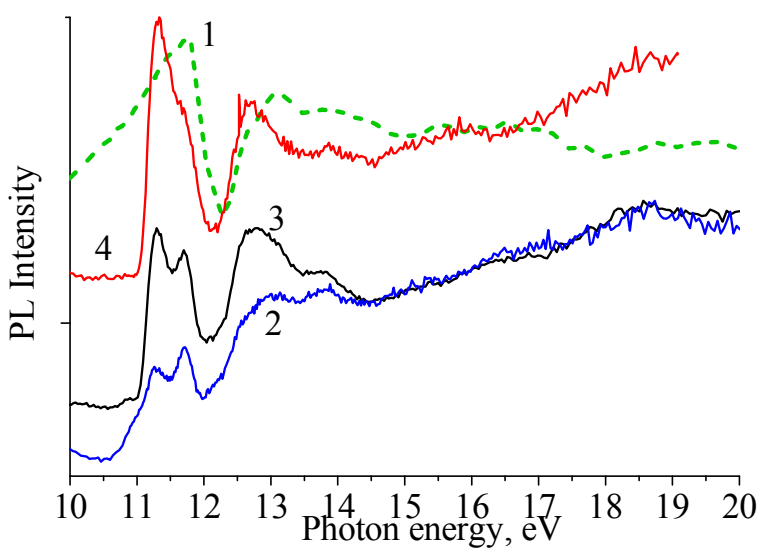

Figure 4. The reflection spectrum of undoped SAF (1) and excitation spectra at $\mathrm{T}=10 \mathrm{~K}$ of intrinsic emission in undoped $\mathrm{SAF} \mathrm{E}_{\mathrm{em}}=3.99 \mathrm{eV}$ (2), Gd:SAF $E_{\text {em }}=3.70 \mathrm{eV}(3)$ : slow component of Ce:SAF $E_{\mathrm{em}}=3.80 \mathrm{eV}$ from [7] (4).

All studied SAF crystals exhibit luminescence at $3.6 \mathrm{eV}$ at $\mathrm{T}=10 \mathrm{~K}$, when excited in the region of excitonic absorption and at higher energies (figure 3, curves 1-3). This band has only microsecond decay components. The corresponding bands found in $[2,11]$ in Pr- and Mn- doped SAF were referred as intrinsic emission, and they are shown for comparison (curves 4,5). Relative intensity of this emission in all studied crystals is low and excitation spectra are similar. Analysis of the luminescence band shows that it is not a single Gaussian peak. Therefore, due to complex nature it can be fit with several bands, peaking at $3.6-4.3 \mathrm{eV}$ One of the possible fits is shown at figure 3 (lower panel). The excitation spectra (figure 4) of this band are similar in all crystals, however, the relative efficiency of 
excitation in band-to band transitions versus excitonic peak is different. In the Ce-doped crystal the Ce emission overlaps with the emission band at $3.6 \mathrm{eV}$, which caused certain difficulties in its interpretation in [7]. The slow component in time-resolved excitation spectrum (curve 4), however, is not influenced by $\mathrm{Ce}^{3+}$ emission, and appears to be very similar to the $3.6 \mathrm{eV}$ band excitation spectrum of other crystals. It is notable that in all these spectra the excitonic peak has a doublet structure.

The XUV-excited $\left(E_{\text {exc }}=120 \mathrm{eV}\right)$ luminescence spectra of all SAF crystals at $\mathrm{T}=10 \mathrm{~K}$ (figure 5) contain one complex broad emission band with dominating microsecond decay components covering the range of $3-5 \mathrm{eV}$. Its spectral components exhibit the same temperature dependence of luminescence without significant changes. Comparing the spectra with those recorded under VUV excitation, one can conclude that the same band at $3.8 \mathrm{eV}$ is present in all spectra, but at XUV excitation the higher energy bands are more intense than at $\mathrm{E}_{\mathrm{exc}}=12.7 \mathrm{eV}$. The small modulations at the spectra of Ce-doped crystals at 4.3-4.5 eV (curve 2) correspond to the $\mathrm{Ce}^{3+}$ emission bands.

While heating, the complex band at $3.8 \mathrm{eV}$ is being quenched, and at $\mathrm{T}=200 \mathrm{~K}$ is not detectable at all. During this quenching process, a new band appears in the range of $5.0-5.5 \mathrm{eV}$ in all studied compounds, having maximum intensity at $\mathrm{T}=150 \mathrm{~K}$ (see figure 7 for Ce:SAF spectra). Its decay curve (figure 8a) contains very fast, sub-nanosecond component. This band can be related to the same center whose emission is observed at $4.5 \mathrm{eV}$ in VUV-excited spectra (figure 1, curve 1), because both bands have rather similar decay curves. The observed difference in their position by $0.5 \mathrm{eV}$ is explained by different spectral sensitivity of detection systems utilized at VUV and XUV setups. Under VUV excitation the ARC spectrometer is used, where the sensitivity is decreasing with increase of photon energy towards UV, while at the XUV setup the Seya monochromator is optimized for VUV.

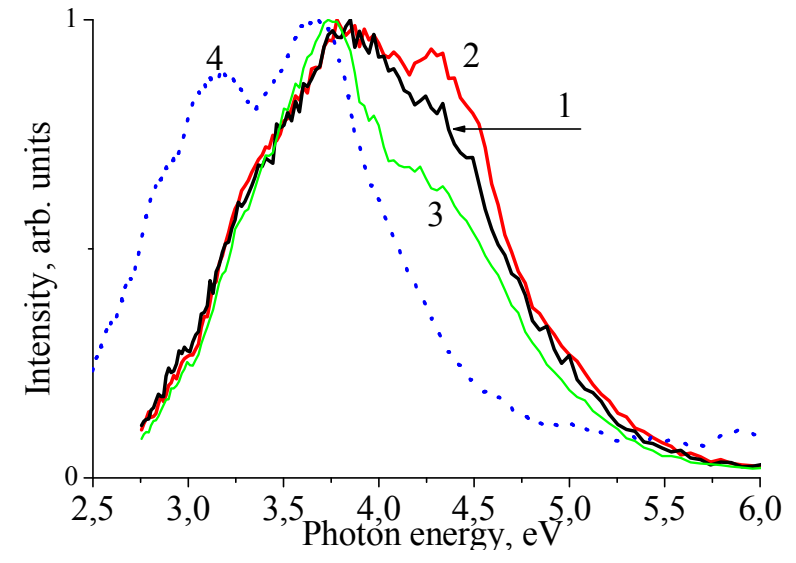

Figure 5. XUV-excited $\left(\mathrm{E}_{\mathrm{exc}}=120 \mathrm{eV}\right)$ emission spectra of SAF crystals at $\mathrm{T}=10 \mathrm{~K}$ : undoped (1), Ce-doped (2) and Gd-doped (3) crystal; For comparison the PL spectra of Gd:SAF at $\mathrm{T}=10 \mathrm{~K} \mathrm{E}_{\mathrm{exc}}=12.7 \mathrm{eV}$ (4)

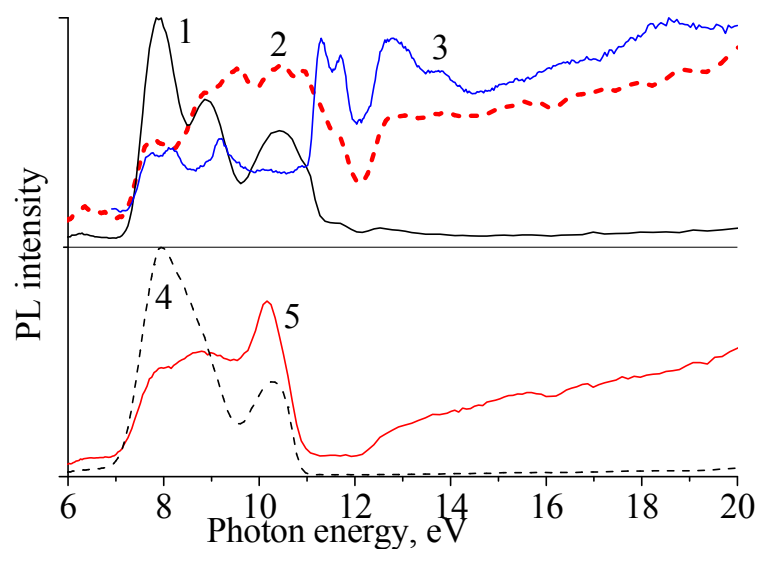

Figure 6. PL excitation spectra of Gd:SAF at $\mathrm{T}=10 \mathrm{~K} \quad \mathrm{E}_{\mathrm{em}}=2.75 \mathrm{eV}(1), \mathrm{E}_{\mathrm{em}}=3.97 \mathrm{eV}$ (2), $\mathrm{E}_{\mathrm{em}}=3.70 \mathrm{eV}(3)$; and at $\mathrm{T}=295 \mathrm{~K} \mathrm{E}_{\mathrm{em}}=2.75 \mathrm{eV}$ (4) and $E_{e m}=3.97 \mathrm{eV}$ (5)

\subsection{The dopant-related emission}

The $\mathrm{Ce}^{3+}$ dopant emission is well discussed in $[7,8]$. The $\mathrm{Ce}^{3+}$ ion substitutes $\mathrm{Sr}^{2+}$ ions in several nonequivalent positions, resulting in several distinct PL centers emitting at different energies in the range 3.8-4.5 eV (see figure 1, curve 2). They can be excited either directly, invoking 4f-5d transitions, or by energy transfer from the above mentioned defects as well as from the electronic excitations of the host.

The detailed studies of temperature dependence of luminescence for Ce-doped SAF crystals are displayed at figure 7 and for decay curves at figure 8. In the temperature range of 10-100 K the shape of spectra remains practically unchanged, but the intensity grows by 3 times while heating from 10 to $50 \mathrm{~K}$. While heating further, quenching occurs, and at $\mathrm{T}=150 \mathrm{~K}$ this wide complex band hardly manifest itself. During this quenching process, the intensity of the fast band at 5.0-5.5 eV grows, and 
the fraction of microsecond components in its decay curve (figure 8a) decreases from dominant to nearly zero. The contribution of $\mathrm{Ce}^{3+}$ fast $5 \mathrm{~d}-4 \mathrm{f}$ emission to the spectra is small and does not change in this temperature region. The decay curves, recorded in one of the $\mathrm{Ce}^{3+}$ emission bands at $4.27 \mathrm{eV}$, show relatively fast ( $\tau=4-5 \mathrm{~ns}$ ) component against the background of slow dominating emission (for comparison, direct Ce excitation [7] has typically $\tau=25-40 \mathrm{~ns}$ ).

Above $150 \mathrm{~K}$, when the fast UV band starts quenching, the intensity of $\mathrm{Ce}^{3+}$ emission decreases simultaneously with this band up to $200 \mathrm{~K}$. In this temperature range microsecond decay components vanish and the dominating component is still quite fast ( $\tau=12 \mathrm{~ns}$, figure $8 \mathrm{~b}$ ). After complete quenching of the fast UV band, the intensity of $\mathrm{Ce}^{3+}$ emission rises up to $300 \mathrm{~K}$, while "usual" $\mathrm{Ce}^{3+}$ decay with $\tau=30 \mathrm{~ns}$ is observed with some slow components present.

The thermostimulated luminescence (TSL) glow curve of Ce:SAF, recorded after electron beam irradiation $\left(\mathrm{E}_{\mathrm{e}}=15 \mathrm{keV}\right)$ is shown in the inset of figure 7. Two complex TSL peaks at $\mathrm{T}=50-60 \mathrm{~K}$ and $\mathrm{T}=180-200 \mathrm{~K}$ were revealed.
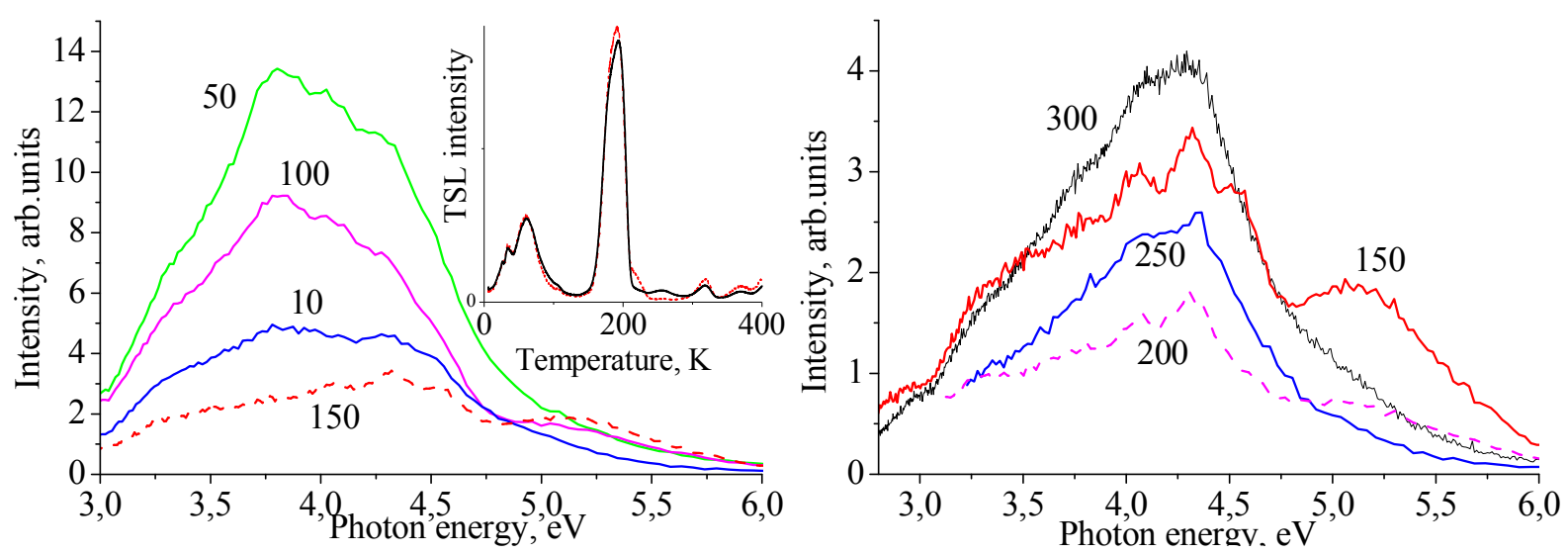

Figure 7. Time-integrated XUV-excited $\left(\mathrm{E}_{\mathrm{exc}}=120 \mathrm{eV}\right)$ emission spectra of Ce:SAF crystals at different temperatures (values indicated on the plots in $\mathrm{K}$ ). Inset: Thermostimulated luminescence glow curves, recorded after irradiation by electron beam $\left(\mathrm{E}_{\mathrm{e}}=15 \mathrm{keV}\right)$. The setup used is described in [12]. Black solid line - integral (1.5-6 eV), red dashed line monitoring $\mathrm{Ce}^{3+}$ emission $(3.75 \mathrm{eV})$

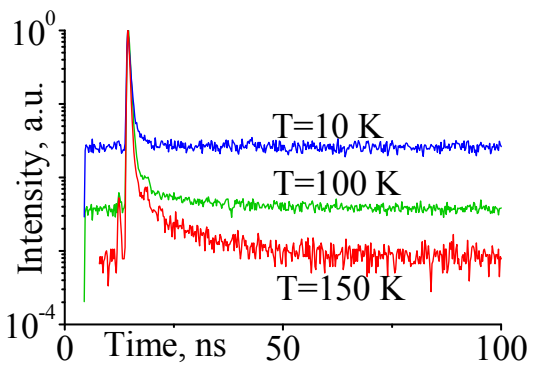

(a)

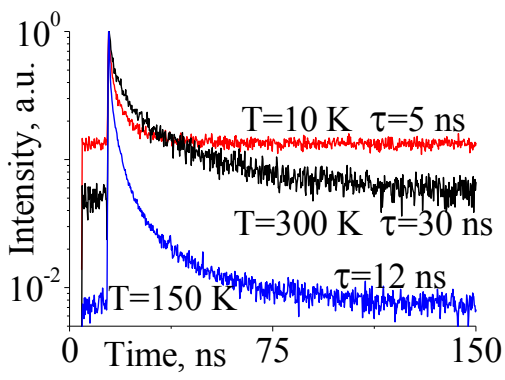

(b)

Figure 8. XUV-excited $(120 \mathrm{eV})$ luminescence decay curves of Ce:SAF crystals at different temperatures: $\mathrm{E}_{\mathrm{em}}=5.25 \mathrm{eV}(\mathrm{a}) ; \mathrm{E}_{\mathrm{em}}=4.27 \mathrm{eV}$ (b). The decay constant of main component is indicated.

The spectroscopy of $\mathrm{Gd}^{3+}$ dopant is rather interesting. Its well-known $3.97 \mathrm{eV}$ line due to the ${ }^{6} \mathrm{P}_{7 / 2}-$ ${ }^{8} \mathrm{~S}_{7 / 2}$ radiative transition in $4 \mathrm{f}^{7}$ configuration is observed in PL spectra, but it cannot be efficiently excited directly through $4 \mathrm{f}-4 \mathrm{f}$ transitions. It appears only at excitation energies higher than $7 \mathrm{eV}$ (figure 6, curves 2, 5). However, figure 1 (curve 4) demonstrates, that while trying to invoke ${ }^{8} \mathrm{~S}_{7 / 2}-{ }^{6} \mathrm{G}$ transitions of the same configuration at around $6.52 \mathrm{eV}$, the other bands appear at 5.6 and at $2 \mathrm{eV}$ (see 
their excitation spectra at figure 2, curve 3 and 4, respectively). These bands with microsecond decay times are not quenched at room temperature. Time-resolved PL spectra show that the excitation in peaks at 6.52 and $9 \mathrm{eV}$ (figure 2, curve 3) result in $5.6 \mathrm{eV}$ emission, while the weak peak at $10.9 \mathrm{eV}$ appears because of overlap with fast defect emission band at $4.5 \mathrm{eV}$.

\section{Discussion}

\subsection{Intrinsic luminescence and self-trapped excitons}

According to [8] the self-trapped exciton emission in pure SAF was not identified earlier. However, STE luminescence was found in Ce:SAF, partly overlapping with $\mathrm{Ce}^{3+}$ emission [7], which caused a problem on its exact spectral positioning. Analyzing the spectral data described above, the band with maximum at $3.6 \mathrm{eV}$ found in all SAF crystals can be assigned with confidence to emission from a triplet state of small radius Frenkel-type STE. Its excitation spectra presented on figure 2 shows the best excitation efficiency for the intrinsic absorption of SAF, while the microsecond-range decay time conclusively supports this assignment. Intrinsic emission is rather weak, especially in the pure crystal, reasons of which are not obvious. In the works $[2,11]$ it is shown that in doped compounds the band assigned to STE can be rather strong, and not totally quenched at RT, while in our case it was not manifest at such high temperature. The probable reason for the observed differences is the presence of lattice defects, which were not mentioned in $[2,11]$. It cannot be fully excluded that in these works the sample preparation method provided powders, which are less influenced of such defects than single crystals. In [2] it was proposed that at higher temperatures, where migration of STE becomes noticeable, they reach dopant ions and resonance energy transfer to Pr ions becomes possible. In our case, high concentration of the growth defects in single crystals can lead to the situation when the probability of STE radiative decay is much smaller than that of interaction with them. The STE energy $(\sim 3.8 \mathrm{eV})$ is not enough to excite growth defects (the first excitation band is $>7 \mathrm{eV}$ ), which lead to a non-radiative decay. However, in doped crystals interaction with rare-earth ions is also possible. The energy of $\mathrm{Ce}^{3+} 4 \mathrm{f}^{0} 5 \mathrm{~d}^{1}$ state is low enough $(3.8-4.5 \mathrm{eV})$ to be excited on interaction with mobile STE, so the TSL peak found in Ce:SAF at 180-200 K (see inset in figure 7), recorded monitoring $\mathrm{Ce}^{3+}$ emission, can arise from this process. In XUV excitation experiment it was found that STE emission is quenched completely at $\mathrm{T}=200 \mathrm{~K}$, which supports this position.

The width and complexity of intrinsic emission bands under XUV excitation cannot be overlooked. It is notable, that under VUV excitation the STE band is not elementary, although its higher-energy components are weak. The possible reason for such complex nature can be found in the SAF crystal structure. There are 20 non-equivalent anion sites providing several non-equivalent positions in the lattice where small-radius self-trapped excitons can be created. The crystal field in these positions can be different, possibly influencing the energy structure of STE. Such model was previously proposed for Ce dopant ions in $[7,8]$. In fluorides small-radius STE is usually treated as a $\mathrm{V}_{\mathrm{k}}$ center with a trapped electron, it is localized in space and affected by influence of neighboring ions. At VUV excitation, due to strong absorption in the excitonic region, only the surface layer is excited, and this may prevent observation of radiative decay of some STE states due to surface losses. XUV radiation has deeper penetration depth providing more favorable conditions for revealing STE spectra (figure 5.)

\subsection{The energy transfer to $\mathrm{Ce}^{3+}$ ions}

The properties of the luminescence of pure and Ce-doped $\mathrm{SrAlF}_{5}$ have been discussed earlier in $[7,8]$. The luminescence bands revealed at 2.9 and $4.5 \mathrm{eV}$ were tentatively assigned to transitions from triplet and singlet excited states of corresponding growth defects. As shown in [7], the efficient excitation of $5 \mathrm{~d}-4 \mathrm{f}$ luminescence of $\mathrm{Ce}^{3+}$ ions in the range of defect absorption $(7-10 \mathrm{eV})$ points to the energy transfer channel from the lattice defects to dopant centers. Resonant energy transfer mechanism was proposed, because in nominally pure SAF crystals the lattice defect emission band at $4.5 \mathrm{eV}$ is well overlapping with the absorption of $\mathrm{Ce}^{3+}$ ions. It is notable that the low intensity of the $4.5 \mathrm{eV}$ band, short decay time and strong temperature quenching point to high probability of non-radiative 
depopulation of the corresponding singlet level. In Ce-doped crystals the resonant energy transfer to the $\mathrm{Ce}^{3+}$ ions is obviously the main depopulation channel, because this process is continuously efficient even at $290 \mathrm{~K}$, while in pure $\mathrm{SrAlF}_{5}$ the $4.5 \mathrm{eV}$ PL band is totally quenched at such high temperature [8]. The character of temperature dependence of XUV-excited luminescence supports our arguments on this energy transfer mechanism. In the temperature region 200-250 K STE emission is already quenched and does not contribute. At the same time, the shorter $(<20 \mathrm{~ns}) \mathrm{Ce}^{3+}$ emission decay time and opposite temperature dependence of $\mathrm{Ce}^{3+}$ emission and fast $\mathrm{UV}$ defect-related luminescence points on their competitive nature. However, the energy transfer between defects and $\mathrm{Ce}^{3+}$ ions may occur in both directions. The lifetime of $\mathrm{Ce}^{3+}$ ions in the excited state is longer than that of the defect (decay times in the case of direct excitation are 25-40 and $3 \mathrm{~ns}$, respectively). The existence of the energy transfer to a quickly decaying center results in the shortening of the decay time for $\mathrm{Ce}(\tau=12$ ns) in comparison with direct excitation. After heating above $200 \mathrm{~K}$, the role of defects is diminished because of thermal destruction or transformation, as the competitive character of the processes disappears. Therefore, at temperatures above $250 \mathrm{~K}$ cerium ions become the dominant PL centers. The slow component in the decay curve can have roots either in the interaction with mobile STE, as described above, or delayed energy transfer by electron-hole pairs, while the fast component can originate from impact excitation by hot photoelectrons as discussed earlier in [13].

\subsection{The energy transfer to $\mathrm{Gd}^{3+}$ ions}

In the vast majority of Gd-containing compounds (see, for example, [14]), $\mathrm{Gd}^{3+}$ ions manifest themselves in PL spectra by the characteristic $3.97 \mathrm{eV}(312 \mathrm{~nm})$ line. This line corresponds to ${ }^{6} \mathrm{P}_{7 / 2}{ }^{-}$ ${ }^{8} \mathrm{~S}_{7 / 2}$ transitions in the $4 \mathrm{f}^{7}$ configuration, and is excited invoking transitions from ${ }^{8} \mathrm{~S}_{7 / 2}$ to higher lying levels of this configuration. In Gd:SAF crystals the situation is rather different. An efficient direct excitation of PL of $\mathrm{Gd}^{3+}$ was not revealed. However, the structure at $6.52 \mathrm{eV}$ in the excitation spectrum of the $5.6 \mathrm{eV}$ band (figure 2, curve 3) exactly matches the energy of ${ }^{8} \mathrm{~S}_{7 / 2}-{ }^{6} \mathrm{G}$ transitions of the $4 \mathrm{f}^{7}$ configuration pointing to effective energy transfer from the dopant to another PL center. The $\mathrm{f}-\mathrm{f}$ transitions are forbidden possessing long lifetimes, and thus the energy transfer to nearby defects or related centers can occur with high probability. The $5.6 \mathrm{eV}$ emission center is practically not excited by any energy transfer mechanisms in the intrinsic absorption of SAF. The PL of such centers was not found in pure and Ce-doped crystals. The nature of the corresponding PL centers probably arises from the charge compensation conditions. As trivalent $\mathrm{Ln}^{3+}$ ions are more likely to substitute $\mathrm{Sr}^{2+}$ [8], an excess charge must be introduced to preserve the crystal lattice neutrality. For $\mathrm{Ce}^{3+}$ the emission band tentatively assigned to charge compensating defects was identified in [8], peaking at $2.7 \mathrm{eV}$. In $\mathrm{Gd}: \mathrm{SAF}$ no such band was found, thus the charge compensation in this crystal is realized in another way. The available compensator can be either interstitial fluorine, or a cation vacancy, or even an $\mathrm{O}^{2-}$ impurity substituting the $\mathrm{F}^{-}$ion. The PL band at 2-2.5 eV (figure 1, curve 4) is more likely to represent the latter case, because its PL and PL excitation spectra are very similar to ones supposed for oxygen impurity in $\mathrm{LiBaAlF}_{6}, \mathrm{LiBaF}_{3}$ and other complex fluorides (see [15] and references therein).

The efficient excitation of the characteristic $\mathrm{Gd}^{3+}$ emission in the range of 7-11 eV points to energy transfer from growth defects to dopants in the same manner as it was proposed for $\mathrm{Ce}^{3+}$ in [7], and this process populates directly the ${ }^{6} \mathrm{P}_{7 / 2}$ level of $\mathrm{Gd}^{3+}$, from which $3.97 \mathrm{eV}$ emission takes place, leaving no possibility for energy drain to the $5.6 \mathrm{eV}$ emission center.

At $\mathrm{T}=10 \mathrm{~K}$, the $\mathrm{Gd}^{3+}$ lines are absent from the emission spectra excited by $11.7 \mathrm{eV}$ photons (figure 3) and in the band-to band transition region (figure 5, curve 4), so the energy transfer to $\mathrm{Gd}^{3+}$ by excitonic and recombination mechanism is inefficient at low temperatures. The rise of intensity under excitation above the band gap (see figure 6, curve 2) is likely due to overlap with the STE emission band. However, at T=290 K, when STE emission is quenched, the excitation spectrum of the $3.97 \mathrm{eV}$ emission shows a similar rise of intensity (figure 6, curve 5), thus recombination mechanism acts at higher temperatures. 


\section{Conclusions}

The luminescence and energy transfer processes in pure and doped $\mathrm{SrAlF}_{5}$ single crystals were investigated by the means of time-resolved UV-VUV-XUV luminescence spectroscopy. Reliable evidence on the STE formation was found. The complex nature of STE emission and excitation bands points to the important role of several non-equivalent sites where excitons can localize. Above $\sim 150 \mathrm{~K}$ the STE emission is quenched due to the increase of their mobility and interaction with defects formed during the crystal growth.

The spectroscopic study of $\mathrm{SrAlF}_{5}$ crystals revealed the role of lattice defects in energy transfer processes to dopant ions at different temperatures. The $\mathrm{PL}$ of $\mathrm{Gd}^{3+}: \mathrm{SrAlF}_{5}$ differs from the majority of other Gd-doped compounds, because no efficient direct excitation of characteristic $3.97 \mathrm{eV}$ luminescence of $\mathrm{Gd}^{3+}$ was found. Instead, the new PL band at $5.6 \mathrm{eV}$ was revealed, which can be excited through higher lying ${ }^{8} \mathrm{~S}_{7 / 2}-{ }^{6} \mathrm{G}$ transitions in $4 \mathrm{f}^{7}$ configuration of $\mathrm{Gd}^{3+}$. This phenomenon is tentatively explained by the high probability of the energy transfer from dopant ions to nearby charge compensating defects. Our results demonstrate that the efficient excitation of the $\mathrm{Gd}^{3+}$ characteristic emission in the range of 7-11 eV is due to energy transfer from the growth defects to $\mathrm{Gd}^{3+}$ (like for $\mathrm{Ce}^{3+}$ ), directly populating the ${ }^{6} \mathrm{P}_{7 / 2}$ level of $\mathrm{Gd}^{3+}$.

\section{Acknowledgements}

This work was supported by RFBR (grant 07-02-00442) and Estonian Science Foundation (grant 8306), the SR studies by European Commission (FP7/2007-2013, 226716). S.I. Omelkov acknowledges gratefully the Archimedes Foundation (Estonia, DoRa5 program) for a stipend supporting his Ph.D. studies. Authors are grateful to E. Feldbach and S. Vielhauer for the help with TSL and XUV studies, numerous discussions and valuable comments on the manuscript.

\section{References}

[1] Villora E G, Shimamura K, Muramatsu K, Takekawa S ,Kitamura K, and Ichinose N $2005 J$. Cryst. Growth 280145

[2] Vink A P, Dorenbos P, de Haas J T M, Donker H, Rodnyi P A, Avanesov A G and van Eijk C W E 2002 J. Phys.: Condens. Matter 148889

[3] Silva E N, Ayala A P, Mendes Filho J, Moreira R L and Gesland J-Y 2004 J. Phys.: Condens. Matter 167511

[4] Kubel F 1998 Zeitschrift fur Anorganische und Allgemeine Chemie 6241481

[5] Mel'nikova S V, Isaenko L I, Gorev M V, Vasil'ev A D and Lobanov S I 2010 Physics of the Solid State $\mathbf{5 2} 509$

[6] Yamaga M, Hayashi E, Kodama N, Itoh K, Yabashi S, Masui Y, Ono S, Sarukura N, Han T P J and Gallagher H G 2006 J. Phys.: Condens. Matter 186033

[7] Omelkov S, Pustovarov V, Kirm M, Ogorodnikov I and Isaenko L 2010 Rad. Meas. 45292

[8] Pustovarov V A, Ogorodnikov I N, Omelkov S I, Cholakh S O and Isaenko L I 2010 Journal of Surface Investigation. X-ray, Synchrotron and Neutron Techniques 4666

[9] Zimmerer G 2007 Rad. Meas. 42859

[10] Kirm M, Lushchik A, Lushchik Ch, Vielhauer S and Zimmerer G 2003 J. Lumin. 102-103 307

[11] van der Kolk E, Dorenbos P, van Eijk C W E, Vink A P, Weil M and Chaminade J P $2004 J$. Appl. Phys 957867

[12] Feldbach E, Kotlov A, Kudryavtseva I, Liblik P, Lushchik A, Maaroos A, Martinson I, Nagirnyi V and Vasil'chenko E 2006 Nucl. Instrum. Methods B 250159

[13] Feldbach E, Kamada M, Kirm M, Lushchik A, Lushchik Ch and Martinson I 1997 Phys. Rev. B 5613908

[14] Ogorodnikov I N, Pustovarov V A, Omel'kov S I, Tolmachev A V and Yavetskii R P 2007 Optics and Spectroscopy 10260

[15] Omelkov S I, Kirm M, Feldbach E, Pustovarov V A, Cholakh S O and Isaenko L I $2010 \mathrm{~J}$. Phys.: Condens. Matter 22295504 